\title{
Volviendo de la diáspora: Liberando un nuevo homo juridicus desde el derecho a la comunicación ${ }^{1}$
}

Returning from the diaspora: Releasing a new bomo juridicus from the right to communication

Retornando da diáspora: Liberando um novo bomo juridicus do direito à comunicação

Revenir de la diaspora: libérer un nouvel bomo juridicus du droit à la communication

从侨民那里回来: 从传播权中释放出一个新的“同性恋”

Sebastián Alejandro García Caicedo ${ }^{2}$

Universidad de Nariño - Colombia

Revista Derechos en Acción ISSN 2525-1678/ e-ISSN 2525-1686

Año 5/N№ 17 Primavera 2020 (21 septiembre a 20 diciembre), 37-54

DOl: https://doi.org/10.24215/25251678e452

Recibido: 01/09/2020

Aprobado: 15/09/2020

1 El presente trabajo hace parte de los primeros esbozos de un proyecto de investigación macro en el curso de una maestría en Derechos humanos, titulado "En disputa por el derecho humano a la comunicación en Colombia. Hacia su fundamentación desde abajo." Así, lo presentado aquí se puede tomar como un avance inicial de una propuesta que acomete construir una fundamentación alternativa del derecho humano a la comunicación desde una postura iusmaterialista.

2 Abogado, Universidad de Nariño. Candidato a especialista en Derecho Constitucional, Universidad Nacional de Colombia. Maestrando en Derechos Humanos - UASLP. Miembro del grupo de trabajo "Crítica jurídica y conflictos socio políticos" de CLACSO. Investigador colaborador en ILSA - Colombia. Teléfono: (+57) 3016411196 - (+52) 551118 0397. Correo electrónico: jito.13@hotmail.com. (ORCID: https://orcid.org/ 0000-0002-3766-9731). 
Resumen: En el presente documento se discute sobre la necesidad de crear un nuevo homo juridicus con carácter emancipatorio y liberador a partir de la fundamentación de un derecho humano a la comunicación que sitúe sus bases filosóficas y de reflexión desde el lugar de las mayorías oprimidas y empobrecidas en el mundo, considerando que aquel es el espacio privilegiado que da verdad para tales fines. Para ello, utilizando lo que nos ofrece el paradigma cualitativo de la investigación, se realiza un ejercicio de pesquisa inscrito en el alcance descriptivo que se reduce a un trabajo de revisión documental y se hace desde un enfoque histórico hermenéutico. Finalmente, se trata de confrontar la hegemonización del discurso, con consecuencias en la identidad, producido por el contexto de neoliberalismo actual, reconociendo que las identidades se dan en contextos determinados, contradictorios, situados y de manera relacional en un marco estructural que las posibilita y disputa de manera constante. En ese orden de ideas, se recurre al concepto del "lugar que da verdad" propuesto por Ignacio Ellacuría en su lucha por la justica y se opta por una concepción materialista de la cultura.

Palabras clave: Identidad, lugar que da verdad, cultura, comunicación, discurso.

Abstract: This document discusses the need to create a new homo juridicus with an emancipatory and liberating character based on the foundation of a human right to communication that places its philosophical and reflection bases from the place of the oppressed and impoverished majorities in the world, considering that it is the privileged space that gives truth for such purposes. To do this, using what the qualitative research paradigm offers us, a research exercise is carried out inscribed in the descriptive scope that is reduced to a work of documentary review and is done from a hermeneutical historical approach. Finally, it is about confronting the hegemonization of discourse, with consequences for identity, produced by the current context of neoliberalism, recognizing that identities occur in specific, contradictory, situated and relational contexts in a structural framework that enables and dispute constantly. In that order of ideas, the concept of the "place that gives truth" proposed by Ignacio Ellacuría in his struggle for justice is used and a materialist conception of culture is chosen.

Keywords: Identity, "place that gives truth", culture, communication, discourse. 
Resumo: Este documento discute a necessidade de se criar um novo homo juridicus de caráter emancipatório e libertador a partir de um direito humano à comunicação que coloque suas bases filosóficas e reflexivas no lugar das maiorias oprimidas e empobrecidas em do mundo, considerando que é o espaço privilegiado que dá verdade para tais fins. Para tanto, a partir do que nos oferece o paradigma da pesquisa qualitativa, realiza-se um exercício de pesquisa inscrito no âmbito descritivo que se reduz a um trabalho de revisão documental e se realiza a partir de uma abordagem histórica hermenêutica. Por fim, trata-se de confrontar a hegemonização do discurso, com consequências identitárias, produzida pelo atual contexto do neoliberalismo, reconhecendo que as identidades ocorrem em contextos específicos, contraditórios, situados e relacionais em um quadro estrutural que possibilita e disputa constantemente. Nessa ordem de ideias, utiliza-se o conceito de "lugar que dá verdade" proposto por Ignacio Ellacuría em sua luta pela justiça e opta-se por uma concepção materialista de cultura.

Palavras-chave: Identidade, "lugar que dá verdade", cultura, comunicação, discurso

Résumé: Ce document discute de la nécessité de créer un nouvel homo juridicus à caractère émancipateur et libérateur basé sur le fondement d'un droit humain à la communication qui place ses bases philosophiques et réflexives de la place des majorités opprimées et appauvries en le monde, considérant que c'est l'espace privilégié qui donne la vérité à ces fins. Pour ce faire, à partir de ce que nous offre le paradigme de la recherche qualitative, un exercice de recherche est mené inscrit dans la portée descriptive qui se réduit à un travail de revue documentaire et se fait à partir d'une approche historique herméneutique. Enfin, il s'agit de confronter l'hégémonisation du discours, avec des conséquences sur l'identité, produite par le contexte actuel du néolibéralisme, en reconnaissant que les identités se produisent dans des contextes spécifiques, contradictoires, situés et relationnels dans un cadre structurel qui permet et dispute constamment. Dans cet ordre d'idées, le concept de «lieu qui donne la vérité» proposé par Ignacio Ellacuría dans sa lutte pour la justice est utilisé et une conception matérialiste de la culture est choisie.

Mot-clés: Identité, «lieu qui donne la vérité», culture, communication, discours. 
摘要：本文件讨论了建立一种具有解放性和解放性的新的同居法的 必要性，该法则基于传播权的基础，该权利的哲学和反思基础来自 被压迫和贫穷的多数派的地位. 这个世界，考虑到正是特权空间为 此目的提供了真理. 为此, 利用定性研究范式为我们提供的内容, 在 描述性范围内进行了一项研究活动，该研究活动被简化为文献审查 工作, 并从诠释历史方法入手. 最后, 它是关于面对新的自由主义当 前语境所产生的话语霸权化，以及对身份的后果，认识到身份是在 一个特定的, 矛盾的, 处境的和关系的语境中发生的, 这种结构性框 架使得和不断发生争执. 按照这种思想顺序, 使用了伊格纳西奥·埃 拉库里亚(Ignacio Ellacuría)在争取正义的斗争中提出的“赋予真 理的地方”的概念, 并选择了唯物主义的文化概念.

关键字: 身份, “提供真理”, 文化, 交流, 话语的地方

\section{Introducción}

Como ya ampliamente se ha discutido en el campo de la antropología, la sociología y algunas corrientes críticas del derecho, este no se reduce al normativismo positivista ni mucho menos puede concebirse como un sistema cerrado, de principios universales e interdependientes y autopoyético. Al contrario, se ha demostrado que el derecho consiste más en una praxis o un discurso de prácticas culturales y sociales como productos históricos que, por eso mismo, es dinámico y no universalizable, dentro de un sistema de relaciones en disputa y dialécticamente configurado. De esa manera, lo que se tiene son procesos culturales conflictivos, donde el derecho es solo una manifestación derivativa de ello, con una articulación relacional dentro del espacio donde se manifiesta, que se traduce en su componente prescriptivo y discursivo. Empero, también tiene un carácter deóntico y performativo en lo que el grupo social considera como lo bueno, lo malo, lo justo, lo injusto y hasta su misma significancia e identidad, delineando aquel concepto discutido dentro de la antropología jurídica del Homo juridicus, como el resultado de algo vivo y proveniente de lo que la misma comunidad acuerda ampliamente. 
En la configuración de esa identidad, existen entonces elementos de asimilación autoconsciente, pero cuyo marco referencial siempre es externo y material: se hace en perspectiva intersubjetiva y dentro de una estructura social amplía que lo posibilita, donde el discurso del derecho está regularmente interviniendo como consecuencia y condición de posibilidad. Hay, por lo tanto, una relación muy marcada entre identidad de los pueblos y los individuos y el derecho. Justamente esta relación es la que se trata de dilucidar muy precariamente en el presente ensayo, haciendo énfasis específicamente en el derecho humano a la comunicación y tratando de aproximarse a una respuesta a los siguientes cuestionamientos: ¿Cuál debería ser el rol del derecho humano a la comunicación en las disputas identitarias de los pueblos? ¿Cómo estructurar un derecho humano a la comunicación desde una praxis que crea identidad en contextos de disputa, con un enfoque relacional, para los oprimidos, víctimas o excluidos?

Sin la intención de ser para nada exhaustivos, se acude entonces a enfocar el análisis posicionándose en lo que Ignacio Ellacuría denomina "el lugar que da verdad" para pensarnos ese derecho humano a la comunicación desde una óptica material, haciéndolo con una mirada que recusa las opresiones múltiples en perspectiva decolonial y lanzando una crítica subyacente al proyecto discursivo de homogeneización propugnado por el capitalismo, que se evidencia más fehacientemente en el contexto actual de globalización y requiere con urgencia la configuración de un nuevo bomo juridicus con enfoque liberador, capaz de edificar una identidad diferente que recupere las ausencias, donde una base materialista de la cultura nos da las luces aproximadas para ello.

\section{Discursos homogéneos y excluyentes}

Como parte de las contradicciones, necesidades y funcionamiento del capitalismo, el mundo y las relaciones humanas se han globalizado. El neoliberalismo, como la manifestación 
cualitativa más latente de esos saltos que se dan con el trasegar histórico, hoy se ha hegemonizado valiéndose de sus sofisticados aparatos, instituciones, dinámicas y formas ideológicas para la alienación necesaria que requiere su círculo de producción y reproducción. Eso ha supuesto que la disputa por los sentidos, la subjetividad y simbología de las personas, se de en planos tanto estructurales como super estructurales, que a su vez ha significado como vencedores a los dueños de los medios de producción y como "vencidos", pero en resistencia inagotable, a los oprimidos, excluidos y empobrecidos por el sentido ontológico y el ethos capitalista. Se ha homogenizado un discurso por antonomasia contradictorio y excluyente, con notables repercusiones en la uniformidad de la cultura y las identidades, como algo requerido para el mismo capitalismo desde su mismo proceso de formación, pues "no hay ninguna cultura popular autónoma, auténtica y completa que esté fuera del campo de fuerza de las relaciones de poder cultural y dominación." (Hall, 2013a, pág. 191)

De manera ineluctable, los más afectados han sido los países del sur global, quienes vendiendo su propio ser importan patrones de conducta y prácticas culturales del norte, por la simple e inconsciente consideración de superioridad de aquella, pauperizando y desconociendo la riqueza, pluralidad y hasta mejor forma de organización y vida social que existe en su propia cultura. Desde el capitalismo se ha exotizado lo externo a su lógica de funcionamiento y necesidades, en aplicación de esa filosofía utilitarista y razón instrumental hegemonizada en el mundo, en una disputa que no solo se da entre dominantes y dominados, sino también al interior de los dominantes para ganar mercados y acumular riqueza. Se trata, como diría Aníbal Quijano, de la pervivencia de la matriz de colonialidad insertada en el ser, saber y poder de los sujetos históricamente colonizados y subordinados por las pretensiones de la modernidad,

3 Hall, S. (2013a). "Notas sobre la deconstrucción de lo popular". En: R., Soto S. (ed.). Discurso y poder en Stuart Hall. Ricardo Soto Sulca editores. Huancayo, Perú. 
pero ampliada a la dialéctica con que opera el capital. Hay un discurso mundializado de la cultura (Wolkmer, 2008) que ha cumplido muy bien con las funciones y características que Foucault (2016) le atribuye al discurso mismo: replantear nuestra voluntad de verdad, su carácter de acontecimiento y borrar la soberanía del significante del otro.

En consecuencia, se tiene un proceso de mundialización cultural inscrito más en las necesidades mercantiles de quienes disputan el poder económico mundial, que en los discursos mismos evidenciados en la pluralidad de conocimientos e informaciones (Moraes, 2013). Por lo tanto, el discurso hegemónico debe comprenderse como una forma de violencia ideológica ejercida sobre las cosas, personas y colectividades, de manera práctica o desde el acontecimiento, siendo ese el lugar donde cada discurso encuentra su regularidad y sus más notables consecuencias. No se debe olvidar que la producción de los discursos en las sociedades viene controlada, seleccionada, distribuida y circulada para su reproducción a partir de procedimientos internamente formulados como marco de contención de poderes exteriores, la aleatoriedad y la misma materialidad (Foucault, 2016).

Se genera una institucionalidad interesadamente inerte, cerrada, mistificada y fetichizada, cuya bandera es verticalmente desconocida y desconocedora, pues el principio de acción e identificación del sujeto individual o colectivo se hace en orden de esas leyes y procedimientos, que durante mucho tiempo han esperado su aparición, pero para honrarlas, pues si consigue algún poder, es únicamente de ella de quien se lo obtiene. Así, el verdadero discurso es aquel normativamente permitido, performativamente adecuado y ritualisticamente conforme. El discurso, por lo tanto, no es solo un mecanismo de traducción de las diferentes luchas y contradicciones presentes en todos los sistemas de dominación, sino aquello por lo que también se lucha y se encuentra en disputa inherente (Foucault, 2016).

Hay una voluntad de verdad en todo discurso que se legitima a partir de esas reglamentaciones y no se encuentra exento 
del deseo y el poder, nublando la visualización de aquella voluntad. El problema es que "la voluntad de verdad que se nos ha impuesto desde hace mucho tiempo es tal que no puede dejar de enmascarar la verdad que quiere." (Foucault, 2016, pág. 24) ${ }^{4}$. Hay en el fondo un interés material representado en formas jurídicas, culturales, ideológicas y organizativas de los espacios de vida del ser humano que, por eso, se convierte en homogéneo y excluyente. ${ }^{5}$ No obstante, eso no significa que sea la única verdad, sino solo una voluntad entre variadas realidades que ha devenido en su hegemonía necesaria para seguir dominando los cuerpos, territorios, mentes, saberes, espacios de apropiación material y simbólica, etcétera. Así, "las formas ideológicas y culturales aparecen simplemente como varias objetivaciones reflexivas de una praxis humana única y no diferenciada, la cual, en las condiciones capitalistas de producción, deviene reificada y alienada (...)" (Hall, 1981, págs. 11-12)

Es por eso que la cultura no puede ser comprendida de una manera aislada y fragmentada, sino más bien como un producto de esas relaciones y macrosistemas económicos y políticos donde confluyen dialécticamente las diferentes formas de asumir y vivir la realidad que, específicamente, se ve inserta en articulaciones de poder y dominación, que para el caso de los dominados ha supuesto en la historia la imposición de formas culturales con efectos identitarios a través de políticas coloniales y neocoloniales (Seider, s.f.). Se trata de un modelo que

\footnotetext{
4 Foucault, M. (2016). El orden del discurso. Editorial Planeta Mexicana, S.A. Ciudad de México, México.

5 Sobre este punto, Grimson (2000) sostiene que cualquier grupo humano y cualquier persona se encuentra, en un contexto espaciotemporal determinado, dentro de un campo de interlocución específico. Un campo de interlocución es un marco dentro del cual ciertos modos de identificación son posibles mientras otros quedan excluidos. Entre los modos posibles de identificación, existe una distribución desigual del poder. (pág. 41).

6 Hall, S. (1981). "La cultura, los medios de comunicación y el efecto ideológico." En: J., Curran, et. Al. (comp.). Sociedad y comunicación de masas. Fondo de cultura económica. Ciudad de México, México.
} 
promueve una visión homogénea del mundo bajo parámetros de estilos de vida que intentan moldear un consumidor universal único que brinde consenso a los intereses de las empresas transnacionales, para lo que necesitan ocultar, ridiculizar e invisibilizar a las identidades culturales locales, especialmente de la periferia global, en un renovado esquema neocolonial. (Maisonnave, 2019, pág. 71$)^{7}$

En ese sentido, hay una atribución antidemocrática y desigual para la distribución y consecuente apropiación y reproducción del discurso: eso se define en un espacio cerrado donde no se discute nada más que las formas de circulación, produciendo indirectamente un marco de no intercambiabilidad y restrictiva de la soberanía de los significantes y narrativas de ese otro discurso exotizado y excluido. Olvida que el ser y estar en el mundo se hace a través de las mediaciones discursivas existentes que están presentes inclusive antes del mismo marco definitorio para la circulación del enmascarado discurso verdadero, siendo que justamente están para encontrar la verdad (Foucault, 2016). Así,

El discurso no es apenas más que la reverberación de una verdad que nace ante sus propios ojos; y cuando todo puede finalmente tomar la forma del discurso, cuando todo puede decirse y cuando puede decirse el discurso a propósito de todo, es porque todas las cosas, habiendo manifestado e intercambiado sus sentidos, pueden volverse a la interioridad silenciosa de la conciencia de sí. (Foucault, 2016, p. 49)

De esa manera, existe un ocultamiento en el supuesto discurso de verdad que no hace más que hegemonizar, segregar

\footnotetext{
7 Maisonnave, M. (2019). El derecho humano a la comunicación. Análisis de la Ley 26.522 (Ley de servicios de comunicación audiovisual) en Argentina (Tesis de Maestría). Universidad Autónoma de San Luis Potosí. San Luis Potosí, México.

8 Foucault, M. (2016). El orden del discurso. Editorial Planeta Mexicana, S.A. Ciudad de México, México.
} 
e impedir el acceso de las mayorías a esa efectiva verdad. Eso significa que existe un lugar privilegiado para encontrarla, más allá de todas las practicas camaleónicas que se surten desde el neoliberalismo en el contexto de globalización cultural, cuyo cometido se delimita no solo en el encuentro consigo mismo, sino también en el develamiento y descubrimiento de lo materialmente real que parece esfumarse y naturalizarse en apariencia. Veamos entonces cuál es ese lugar.

\section{El lugar que da verdad y liberación}

Dentro de los planteamientos filosóficos de Ignacio Ellacuría de la realidad histórica como el último estadio de la realidad, recuerda el carácter liberador de las acciones de los sujetos como eso siempre presente en la praxis socio histórica ${ }^{9}$. Pero no se trata de cualquier sujeto, sino de aquel que aún no ha logrado liberarse a pesar de su capacidad creativa. Se trata de un sujeto sometido al dinamismo de su materialidad y realidad, así como al proceso histórico evolutivo de su existencia que, a su vez, determina sus posibilidades progresivas de liberación explicitada por imbricaciones de lo anterior y lo posterior, de lo inferior y lo superior y de su propia materialidad. ${ }^{10}$ La liber-

9 Valga recordar que, para Ellacuría, la realidad histórica es el objeto de la filosofía, entendida como metafísica intramundana, no solo por su carácter globalizante y totalizador, sino en tanto realidad histórica: campo máximo de lo real (respectividad de la realidad en el contexto del dinamismo), que supone un momento de realidad que se forma por todos los modos de realidad en respectividad y contexto dinámico. En ese contexto, la historia es un constante de ir hacia nuevas formas de realidad, en donde la interacción de las cosas forma las diversas formas de realidad, produciendo modos de realidad en estadios no jerárquica sino de apropiación que hace que algunos sean más o menos realidad (Ellacuría, 1999).

Al respecto se puede ver: Ellacuría, I. (1999). Filosofía de la realidad histórica. UCA editores. San Salvador, El Salvador.

10 Con esto se supera grados de abstracción e idealismo en la construcción de libertad humana, muy propios en la filosofía occidental clásica liberal y conservadora y se muestra la cercanía de Ellacuría con el marxismo y el materialismo -sin caer en el determinismo económico-, así como en la noción de realidad de Zubiri. Afirma Rosillo (2013): “La libertad en abstracto no existe; lo existente son las acciones libres, las cuales hacen del ser humano un ser relativamente absoluto, que puede optar dentro de una serie de posibilidades en virtud 
tad es, entonces, un proceso de liberación que se da de, en y desde la naturaleza sumergida en dinamismos para ser lo que se quiere ser, erigiéndose como presupuesto y consecuencia de la misma historia. "Sin el proceso de liberación básico no habría historia, ni novedad en ella, y es gracias a ella que aparece un proceso de praxis en el ser humano con base en el dinamismo de actualización de posibilidades." (Rosillo, 2013, pág. 102) ${ }^{11}$

Las anteriores afirmaciones, coligen la necesidad de que sea el ser humano el responsable de su liberación y, por lo tanto, de la creación de su propia historia, cuya libertad como finalidad se obtiene solo si esos ejercicios de responsabilidad de liberación se surten. El sujeto histórico práxico Ellacuriano, es entonces, auto responsablemente transformador y liberador, donde su materialidad, conciencia y ejercicio intelectivo de su realidad delinean su práctica activa, siempre como maniobras tendientes a producir estructuras más humanas. Empero, se trata de un ejercicio dialéctico, contradictorio y de disputa donde no siempre se sale triunfador. Es decir, puede que el sujeto práxico no logre construir su libertad a pesar de sus prácticas de liberación.

En específico, Ellacuría considera que la realidad del derecho y las disputas en torno a su configuración y fundamento debe darse desde el empobrecido, el oprimido que lucha por superar la alineación proveniente desde los discursos excluyentes o la totalidad alienante (Rosillo, 2013). Para él, ese es el lugar que da verdad, siendo que no se encuentra intrínsecamente en su logos, sino más bien en su realidad material como mayorías populares. Es decir, la verdad se encuentra no en lo que se dice de la injusticia, sino en la injusticia misma, que se encarna en este sujeto de derechos humanos. "A lo que apunta

\footnotetext{
de su apertura a la realidad." (pág. 101) De ahí que se pueda afirmar que la construcción filosófica de libertad en Ellacuría supere análisis socio políticos ya dados y se haga desde el inicio de la misma realidad.

11 Rosillo, A. (2013). Fundamentación de los derechos humanos desde América Latina. Editorial Ítaca. México.
} 
Ellacuría como crítica es al proyecto de la recta estructuración del sistema de posibilidades que se muestra como injusto en las mayorías populares excluidas y no a la recta estructuración de los conceptos". (Leal, 2015, pág. 62) ${ }^{12}$

Se tiene así, que el sujeto sobre quien recae la acción práxica, así como el derecho desde esta fundamentación es un sujeto amplío, diversificado y con carácter comunitario. No se trata de otorgar derechos y exigir responsabilidades tan solo a individuos o comunidades, sino de superar esa línea divisoria y entender al sujeto en función dialéctica y articulada entre el yo y el todo(s), que, por eso, le otorga responsabilidad comunitaria de sentido y de acción. Asimismo, no se trata de un sujeto abstracto, sino concreto cuyo horizonte de acción se evidencia específicamente en el contexto donde actúa: es un sujeto en, desde y para la realidad histórica con función liberadora inexorable.

Por demás, se trata de un sujeto que entiende la relación entre el derecho con el bien común y la justicia, que precisamente surge para procurar estos últimos y superar el mal común existente, cuyo núcleo de relación se evidencia en su condición de víctimas de la estructura, imposibilidades de libertad, opresión y negación de su ser y hacer (López, 2015). Es un sujeto, que a la muerte le opone la vida e irrumpe para modificar la realidad; que pasa de un logos meramente contemplativo, ahistórico y especulativo a uno de praxis socio histórica.

\section{Sobre el derecho humano a la comunicación: Identidad desde abajo y materialidad}

Como se pudo puntuar en páginas previas, el surgimiento de nuevas formas de dominación y exclusión producidas por

12 Leal A., R. (2015). "Estructura e historia en Ellacuría. Hacia una aproximación hermenéutica a "La filosofía de la realidad histórica" a partir de la filosofía de Xavier Zubiri". En Alejandro Rosillo M. \& Ramón M. Pérez M. (Coord.), Historizar la justicia. Estudios sobre el pensamiento de Ignacio Ellacuría (pp. 31-64). Centro de Estudios Jurídicos y Sociales Mispát, A.C. \& Universidad Autónoma de San Luis Potosí. San Luis Potosí, México. 
el discurso de la globalización y el neoliberalismo ha tenido notables consecuencias en las practicas culturales, identidades y normas de la condición de vida de la sociedad a escala planetaria. De la misma manera, esa estructura tiene en su interior, lógica y necesidades un sujeto al que se le ha desconocido su identidad y potencialidad, pero que contiene en sí mismo el espacio propicio para encontrar la verdad del sistema y luchar por su liberación: los oprimidos o mayorías populares. Tendría cabida preguntarse entonces, ¿cuál puede ser la fundamentación del derecho humano a la comunicación en ese contexto, en relación con la generación de una disputa cultural e identidad para esas mayorías populares?

Para pensarse eso, se hace inevitable comprender que el derecho humano a la comunicación debe necesariamente entender que el sujeto sobre el que puede operar es un sujeto vivo, corporal, con necesidades materiales, intersubjetivo y en una constante actividad práxica. Se debe superar esa visión abstracta, monológica, contemplativa y etnocéntrica que la modernidad y el capitalismo tienen del sujeto. Eso, como un punto de partida para dotar de sentido y, en especial, de materialidad y contexto tanto al sujeto como al mismo derecho humano a la comunicación. Se tiene entonces una visión antropológica del derecho compleja y ampliada que reinterpreta las realidades normativas en las comunidades desde una perspectiva cultura concreta y material (Rosillo, 2019).

Hay un uso desideologizado y superador de la formalidad opresora del sistema en ello, pues dicha materialidad comprende la finalidad del contenido del acto humano; es decir, entiende que la finalidad de vivir es estar vivo y que para ello es necesario satisfacer las necesidades materiales de existencia. ${ }^{13}$ Desde allí

\footnotetext{
13 Sobre este punto, huelga recordar que, en esa satisfacción de necesidades, el sujeto tiene cierto margen de definición, pero limitado. En otras palabras, existe una materialidad en la historia que ata al sujeto a su circuito natural de la vida humana como la condición de posibilidad para la propia vida (Rosillo, 2019). De ahí que sea necesario pensarse los actos humanos de una manera más consciente y complejizada.
} 
se brega porque existan las condiciones mínimas para la producción, reproducción y aumento de la vida humana, pues la vida no es un fin utilitario en sí mismo que se somete a la racionalidad de la competencia, sino que es el contenido mismo de la vida. Hay un compromiso del derecho humano a la comunicación con ello, si no quiere quedarse en planos ideales y meramente de libertad en su fundamentación, donde se hace necesario "que a través de un principio material la satisfacción de necesidades se constituya como un hecho radical." (Rosillo, 2019, pág. 11) ${ }^{14}$

De esa manera, se construye un parámetro para actuar que, además, reconoce la mediación necesaria para la vida que supone el acto de comunicarse. No se trata de solo ser libre para expresar, informar o dar noticias, sino de reconocerse como sujeto comunicante, relacional e intersubjetivo que encuentra el desarrollo de la producción y reproducción de su vida en la alteridad con el otro. "El sujeto se hace sujeto por la afirmación de su vida, pero esta subjetividad se complementa con la afirmación de la vida del otro. El otro aparece con claridad en las crisis de los sistemas que causan muerte." (Rosillo, 2019, pág. 23) ${ }^{15}$. Es un hecho radical de interpelación y confrontación material a la imposibilidad de la vida, donde el oprimido refuta materialmente la verdad del sistema que origina esa negación.

No se desconoce la dimensión individual del sujeto, sino que se complejiza, contextualiza y materialmente se llena de contenido, "para generar transformaciones en el sistema buscando que sus estructuras sean capaces de satisfacer las necesidades para la producción, reproducción y desarrollo de la vida."

14 Rosillo, A. (2019). "Materialidad de derechos humanos y antropología jurídica". En: G., Luevano; A., Terven \& A., Rosillo. (coord..). Prácticas institucionales de la justicia estatal y comunitaria. Estudios de antropología jurídica (pp. 9-30). Centro de Estudios Jurídicos y Sociales Mispát, A.C. \& Universidad Autónoma de San Luis Potosí. San Luis Potosí, México.

15 Rosillo, A. (2019). "Materialidad de derechos humanos y antropología jurídica". En: G., Luevano; A., Terven \& A., Rosillo. (coord..). Prácticas institucionales de la justicia estatal y comunitaria. Estudios de antropología jurídica (pp. 9-30). Centro de Estudios Jurídicos y Sociales Mispát, A.C. \& Universidad Autónoma de San Luis Potosí. San Luis Potosí, México. 
(Rosillo, 2019, pág. 11) ${ }^{16}$. Por demás, es un argumento que integra una praxis que busca liberar al sujeto dueño de la verdad como momento material, objetivo e intelectivo situado en las victimas que busca la transformación del estado de cosas que lo ha puesto en esa posición y genera una nueva institucionalidad que posibilita la vida e impide la muerte. Se tendría un derecho humano que no niega alteridades, se ubica desde la exterioridad y se expresa como juridificación de necesidades vivas y legitimas y no meros deseos como voluntad de verdad. Con ello coadyuva para que los oprimidos, excluidos y víctimas del sistema luchen desde esta esfera en la producción, circulación y reproducción de un sentido común, simbología y discurso que enseñe que primero se deben satisfacer necesidades materiales de existencia. Funge, a su vez, como un instrumento de lucha para superar las causas que originan la negación y opresión mismas, con el fin de que la vida se aumente en la comunidad (Rosillo, 2019).

Con lo anterior tampoco se niega el carácter contextual que debe tener este derecho humano; por el contrario, se reafirma en su función de creación identitaria y formación cultural de los sujetos, pero lo hace desde un enfoque materialmente liberador. No se reduce a una simple armonización horizontal de las relaciones de poder, sino que se afirma posicionadamente con las victimas en esa dialéctica configurativa de las formas de ser y estar en el mundo. Acoge esa provocante preocupación que comparte Octavio Paz (2019) sobre las recurrentes preguntas de los pueblos sobre su ser y los medios para ser eso que dicen ser, confrontando la idea de que solo el Estado tiene capacidades de regular la producción de subjetividad, en un entramado sistémico capaz de definir identidades y verdades. Sin duda, juega un papel importante, pero ya existe una subjetividad formada $y$

16 Rosillo, A. (2019). "Materialidad de derechos humanos y antropología jurídica". En: G., Luevano; A., Terven \& A., Rosillo. (coord..). Prácticas institucionales de la justicia estatal y comunitaria. Estudios de antropología jurídica (pp. 9-30). Centro de Estudios Jurídicos y Sociales Mispát, A.C. \& Universidad Autónoma de San Luis Potosí. San Luis Potosí, México. 
en formación que es exaltada a través de un derecho humano a la comunicación con estas características en un contexto donde la identidad es disputada. Al efecto, no se debe olvidar que "las identidades siempre son construcciones sociales que se producen y reproducen -a veces estratégicamente- en contextos históricos de intereses encontrados." (Baronnet, Mora \& Stahler-Sholk, 2011, pág. 32) ${ }^{17}$

Con ello, se confirma el carácter relacional, histórico, dinámico y conflictivo que supone el campo de la cultura y la identidad, donde emerge un sujeto político capaz de resignificar narrativas en confrontación directa contra las estructuras que auspician la dominación y explotación. Los medios de comunicación ya no serán parte de una industria cultural ajustada a las preferencias discursivas dominantes (Hall, 2013a). Y no es un mero ejercicio de contracultura ni cultura contrahegemónica, sino de ejercicio práxico cultural en sí mismo que se define por el criterio de la producción y reproducción de la vida desde los sujetos empobrecidos que a través de él develan la verdad, se reconocen y crean nuevos sentidos identitarios y simbologías para liberarse, con consecuencias en sus prácticas y formas de relacionamiento. Discuten sobre nuevos mensajes, ponen en forma de acontecimiento su soberanía y de manera autoconsciente dilucidan sobre la necesidad de que, para modelar la efectivización de su apuesta, inexorablemente deben contraponerse al sistema de dominación que ha inoculado en sus consciencias sus intereses (Hall, 2013b).

En definitiva, un derecho humano a la comunicación con esas características discutiría sobre "las posibilidades de una nueva cultura jurídica con su legitimación asentada en el reconocimiento de la justa satisfacción de necesidades básicas y en la acción participativa de los sujetos insurgentes, singulares y colectivos". (Wolkmer, 2008, p. 210) que, en el marco de

17 Baronnet, B., Mora, M.\& Stahler-Sholk, R. (Coord.). (2011). Luchas "muy otras". Zapatismo y autonomía en las comunidades indígenas de Chiapas. UAM, CIESAS, Universidad Autónoma de Chiapas. México. 
creación identitaria a partir de una praxis relacional, se ubica para que aquella sea liberadora y no reproductora de estructuras y formas de dominación. No solo se enmarca dentro un uso desideologizador y que desfetichiza la relación sujeto-derecho, sino que propugna para que aquel "lugar que da verdad" emerja como alternativa viva de sociabilidad y se haga visible en su praxis concreta invisibilizada por los discursos homogéneos y excluyentes del capitalismo, potenciando su capacidad creadora de la historia.

\section{Conclusiones}

Las dinámicas propias del capitalismo han supuesto una realidad compleja que se ha extendido a escala global. Esa realidad, se construye a partir de ciertos discursos unívocos que se legitiman como pretensiones de verdad a partir de diversos mecanismos, con efectos en las consciencias, identidades, instituciones y formas de ser, tener y actuar en la sociedad. Los intereses de la clase dominante se representan en ello, al punto de hablar de una mundialización de la cultura, que, por las necesidades del capital, no puede admitir más diferencias que las mismas creadas y admitidas por el mercado. Hay una subsunción del sujeto en toda su dimensión a esa lógica, que lo enajena y absorbe en un modo de vida ficticio.

Se habla entonces de que hay una falsa verdad en la realidad y una aparente identidad de los sujetos y comunidades, en un contexto de disputa, hostil y conflictivo que predetermina la praxis de esa creación identitaria. En ese espacio toma relevancia la comunicación, puesto que por ahí es por donde más efectivo se hace el discurso de homogenización y exclusión que se abre paso fácil e impunemente por las normas burguesas. En ese sentido, se hace necesario pensar un derecho humano a la comunicación desde una óptica realmente verdadera; es decir, desde el locus que tiene la verdad por su propia realidad como víctimas de esa exclusión sistemática inherente. Para ello, uno de los principios esenciales para su fundamento es la materialidad 
o la necesidad de exaltar la producción y reproducción de la vida como el punto inicial que funja como una deriva en una construcción liberadora de la identidad en su contenido real, material y práxico de configuración.

Como un "sueño lírico de un discurso que renaciese absolutamente nuevo e inocente en cada uno de sus puntos y que reapareciese sin cesar, en toda su frescura, partiendo de los sentimientos, de los pensamientos o de las cosas." (Foucault, 2016, pág. 27) ${ }^{18}$, el lugar que da verdad propuesto por Ellacuría es el espacio perfecto para definir un derecho humano a la comunicación que, entendiendo a la identidad dentro de un marco de relaciones sociales localizadas e históricas, constituye un sujeto emergente, guiado por la dignidad humana, la vida y la diversidad, reconociendo el poder de acción de la comunidad y el retorno de agentes históricos que pelean por su existencia, superando monismos formales universalistas y alienantes procurados por el derecho moderno.

En consecuencia, se trata de un derecho que no solo reconoce identidades y diferencias, sino que propugna por la construcción de una forma de ser verdaderamente liberadora. Se formula, entonces un derecho humano a la comunicación desde un lugar que disputa los sentidos de las categorías identitarias dominantes y las categorías mismas, pues "resignificar otro modo de vida empuja la dimensión cultural por otras modalidades de experiencia, de relaciones sociales y ordenaciones de las practicas emergentes e instituidas" (Wolkmer, 2008, pág. 205) ${ }^{19}$.

\footnotetext{
18 Foucault, M. (2016). El orden del discurso. Editorial Planeta Mexicana, S.A. Ciudad de México, México.

19 Wolkmer, A. (2008). "Mundialización cultural, pluralismo jurídico y derechos humanos". En: A., Rosillo (coord.). Derechos humanos, pensamiento crítico y pluralismo jurídico (pp. 205216). Centro de Estudios Jurídicos y Sociales Mispát, A.C. \& Universidad Autónoma de San Luis Potosí. San Luis Potosí, México.
} 